\title{
SPATIAL LOCALIZATION OF MODELLED OBJECTS OF REVOLUTION IN MONOCULAR PERSPECTIVE VISION
}

\author{
M.DHOME, J.T.LAPRESTE, G.RIVES \& M.RICHETIN \\ Electronics Laboratory, UA 830 of the CNRS \\ Blaise Pascal University of Clermont-Ferrand \\ 63177 AUBIERE CEDEX (FRANCE)
}

\author{
tel: 73.40.72.28; fax : 73.40.72.62 \\ email : EVALEC@ @RMOP11.BITNET
}

\section{INTRODUCTION}

In monocular vision, recognition and spatial localization are often difficult problems because the appearance of the observed objects varies according to the point of view. The various aspects of an object can be radically different as shown in Figure 1.

For polyhedral objects, i.e. the block-world, many works have been done on the labelling of the visible edges and on the interpretation of the contours in terms of shape, of relative positions of plane facets, deduced from this labelling at the intersection of the edges [SHI-87]. Moreover it has been proved that localization of modelled polyhedra can be obtained from one single image [DHO-89].

For curved objects, the contours which can be detected in an image and which result from different physical phenomena in the image formation process, are curves whose geometrical characteristics depend on the shape of the object. They are the projections of space curves whose position on the surface of the object is related with the point of view.

For objects of revolution, much information can be derived from a single perspective view, especially their localization in the viewer coordinate system.

Figure 1 presents differents aspects of the image of a vase. The shape of the contours which could be extracted would be very variable from one image to an other. But if we look at the external contours of such an object, i.e. the contours which separate the vase from the background, it is worth noting that they are of two different kinds :

- either they come from the perspective projection of angular edges on the object surface which are obviously circles,

- or they are the projections of limbs i.e. of space curves on the object surface at each point of them the normal of the surface is orthogonal to the viewing direction of this point.

On figure 1, the recurrence of some shape or special points is noticeable in the external contour : ellipses, zero-curvature points, angular points. These three geometrical features are very useful to solve the localization problem as it will be shown in the next sections.

For modelled curved objects, especially generalized cylinders, J. Ponce [PON-89b] has recently proposed a very general algebraic approach for recognition and localization. He made successful experiments on objects of revolution. But we believe that it will be always valuable to exploit geometrical properties of the contours of an object 
since we will see that the resolution of the localization problem is quite simple in this way. Moreover this geometrical approach gives a deep understanding of the perspective projection of curves and surfaces.
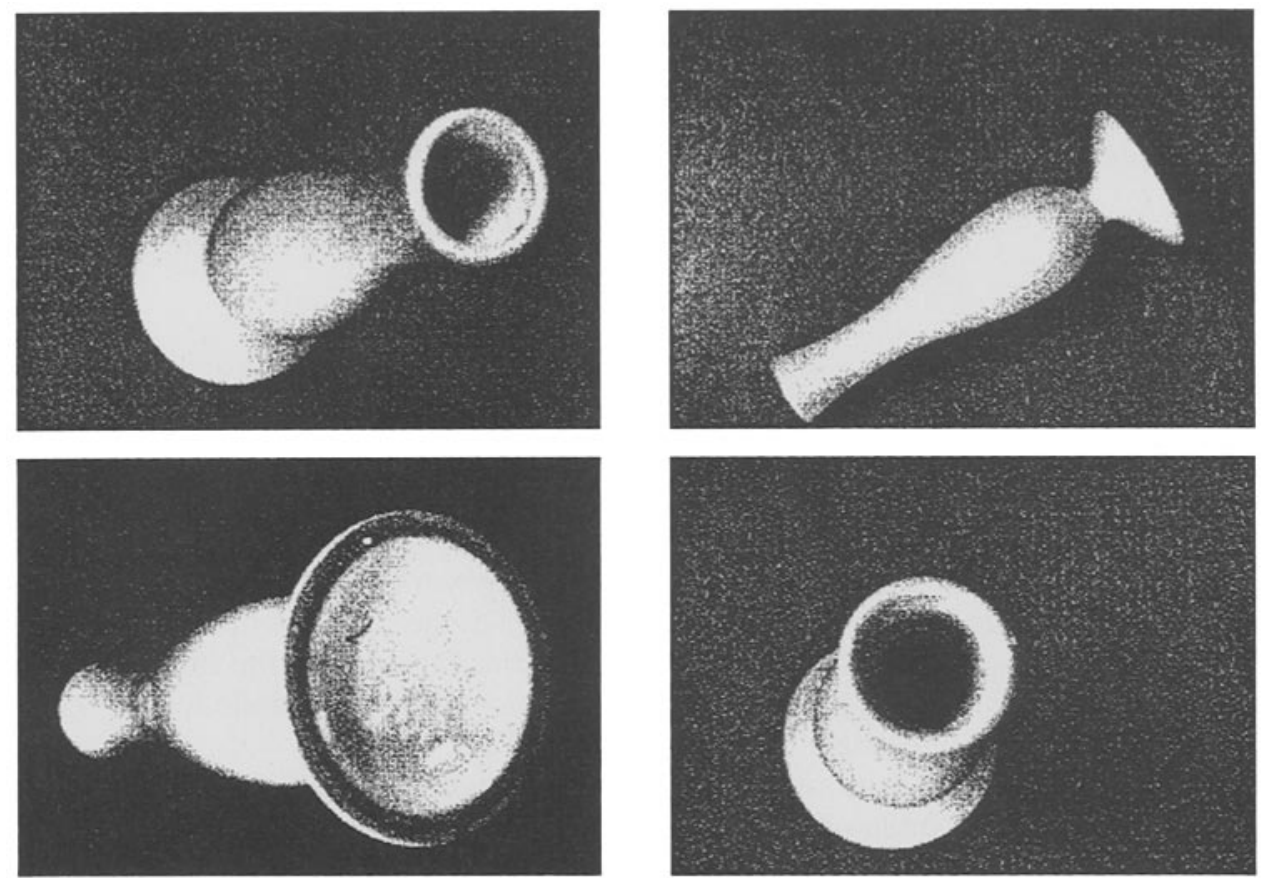

Figure 1. Various aspects of the image of a vase.

\section{INTERPRETATION OF ELLIPTICAL CONTOURS}

\subsection{The cyclic plane problem}

Let us consider an object of revolution having a circle as an angular edge, for which the support plane, the center and the radius are known in an object coordinate system. Let us suppose also that the perspective projection of this circle is viewed in the image. From this projection, which is an ellipse in the general case, it is possible to recover the spatial attitude of the object.

If the ellipse has been estimated by the following equation,

$$
a_{0} x^{2}+b_{0} x y+c_{0} y^{2}+a_{0} x+e_{0} y+1=0
$$

the equation of the cone defined by the optical center of the camera and by the viewing directions of all the points of the ellipse is :

$$
a_{0} x^{2}+b_{0} x y+c_{0} y^{2}+\frac{d_{0}}{f} x z+\frac{e_{0}}{f} y z+\frac{1}{f^{2}} z^{2}=0
$$

with $f$ being the focal length of the camera.

The location problem is then a cyclic plane problem, a cyclic plane being a plane whose intersection with a cone is a circle. Moreover this circle must have a given radius here. 


\subsection{Determining the cyclic planes}

In order to simplify this determination, it is useful to choose a viewer coordinate system such that the equation of the cone is reduced to :

$$
\frac{x_{1}^{2}}{a_{1}^{2}}+\frac{y_{1}^{2}}{b_{1}^{2}}-\frac{z_{1}^{2}}{f^{2}}=0 \quad \text { with } \quad \frac{1}{a_{1}^{2}} \geq \frac{1}{b_{1}^{2}}
$$

This new coordinate system is obtained after a rotation $R_{D}$ of the original one which makes diagonal the following matrix :

$$
\left(\begin{array}{ccc}
a_{0} & \frac{b_{0}}{2} & \frac{d_{0}}{2 f} \\
\frac{b_{0}}{2} & c_{0} & \frac{e_{0}}{2 f} \\
\frac{d_{0}}{2 f} & \frac{e_{0}}{2 f} & \frac{1}{f^{2}}
\end{array}\right)
$$

In this coordinate system, the great axis of the ellipse can always be horizontal ( $X$ axis). To solve the cyclic plane problem, then it is sufficient to find the rotation $R_{\alpha}$ of angle $\alpha$ around the horizontal axis for which the intersection of the cone by a vertical plane is a circle.

After the application of rotation $R_{\alpha}$, the equation of the cone becomes :

$\frac{x_{1}^{2}}{a_{1}^{2}}+\left(\frac{\cos ^{2} \alpha}{b_{1}^{2}}-\frac{\sin ^{2} \alpha}{f^{2}}\right) y_{1}^{2}+\left(\frac{\sin ^{2} \alpha}{b_{1}^{2}}-\frac{\cos ^{2} \alpha}{f^{2}}\right) z_{1}^{2}+2\left(\frac{\sin \alpha \cos \alpha}{b_{1}^{2}}+\frac{\sin \alpha \cos \alpha}{f^{2}}\right) y_{1} z_{1}=0$

The intersection of the cone by a vertical plane (constant $z_{1}$ ) is a circle if and only if the coefficients of $x_{1}^{2}$ and $y_{1}^{2}$ are equal. This gives equation (4):

$$
\frac{1}{a_{1}^{2}}=\frac{\cos ^{2} \alpha}{b_{1}^{2}}-\frac{\sin ^{2} \alpha}{f^{2}}
$$

which implies the following determinations for $\cos \alpha$ and $\sin \alpha$ :

$$
\cos ^{2} \alpha=\frac{b_{1}^{2}\left(a_{1}^{2}+f^{2}\right)}{\alpha_{1}^{2}\left(b_{1}^{2}+f^{2}\right)} \quad \sin ^{2} \alpha=\frac{f^{2}\left(a_{1}^{2}-b_{1}^{2}\right)}{a_{1}^{2}\left(b_{1}^{2}+f^{2}\right)}
$$

and a multiple determination for angle $\alpha$ :

$$
\alpha_{1}, \quad \alpha_{2}=\pi-\alpha_{1}, \quad \alpha_{3}=\pi+\alpha_{1}, \quad \alpha_{4}=-\alpha_{1} .
$$

A quadratic cone has then two families of cyclic planes. For any plane of each family there are two positions of the object since it can placed in each of the two half spaces delimited by this plane. Then there are four families of positions of the object.

To solve the localization problem completely, the center of the circle has to be determined. Thus four spatial attitudes for the object will be got.

\subsection{Finding the center of the circle}

From equations ( 3 ) and (4), the cone equation is :

$$
x_{1}^{2}+y_{1}^{2}+a_{1}^{2}\left(\frac{1}{b_{1}^{2}}-\frac{1}{a_{1}^{2}}-\frac{1}{f^{2}}\right) z_{1}^{2} \pm 2 \frac{\sqrt{a_{1}^{2}+f^{2}} \sqrt{a_{1}^{2}-b_{1}^{2}}}{b_{1} f} y_{1} z_{1}=0
$$

The symbol \pm corresponds with the sign of the product $\cos \cdot \sin$ for the considered angle $\alpha_{i}$. 
As the space position of a circle of radius $\rho$ must compatible with the cone equation, this circle must be in a vertical plane and its center $C$ must be in the plane $\left(y_{1}, O, z_{1}\right)$. The coordinates of $C$ are then of the form $\left(0, y_{1_{c}}, z_{1_{c}}\right)$ which corresponds with the following cone equation:

$$
x_{1}^{2}+y_{1}^{2}-\frac{\rho^{2}+y_{1 c}^{2}}{z_{l_{c}}^{2}} z_{1}^{2}+2 \frac{y_{1_{c}}}{z_{1_{c}}} y_{1} z_{1}=0
$$

The coordinates of $C$ are derived from equations (5) and (6).

The sign \pm has the same meaning as in equation (5).

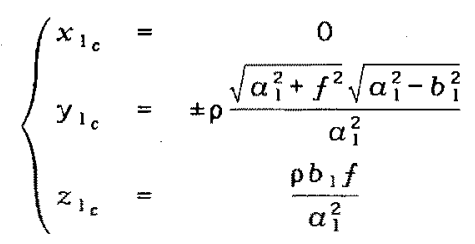

The position of the circle center $C$ in the original viewer coordinate system is obtained by applying the rotation $R_{\alpha_{t}}^{-1}$ and the rotation $R_{D}^{-1}$.

\subsection{Experiment}

The preceding method allows to calculate the four spatial attitudes of a given radius circle defined by the position of its center and by the normal to its support plane, which is compatible with an elliptical contour. Knowing the position of a circular edge on the surface of an object of revolution, it is then straightforward to determine the rigid transform to apply to the object model to bring it in position such that the projection of the circular edge will give the observed elliptical contour.

Figure 2 (left) shows the detected contours of a brightness image of a vase. One of the detected ellipses appears in figure 2 (right). Figure 3 presents the four attitudes of the object model which have been determined from the interpretation of this ellipse.

It is quite visible that three of them are not valid. The quality of the covering of the external contours of the projected model image by the contours of figure 2 (left) can be evaluated [RIC-89]. A score between 0 and 1 is calculated (see figure 3) and the selected solution has the highest score. Both the brightness image and the projected model image for the selected solution are presented in figure 4 .
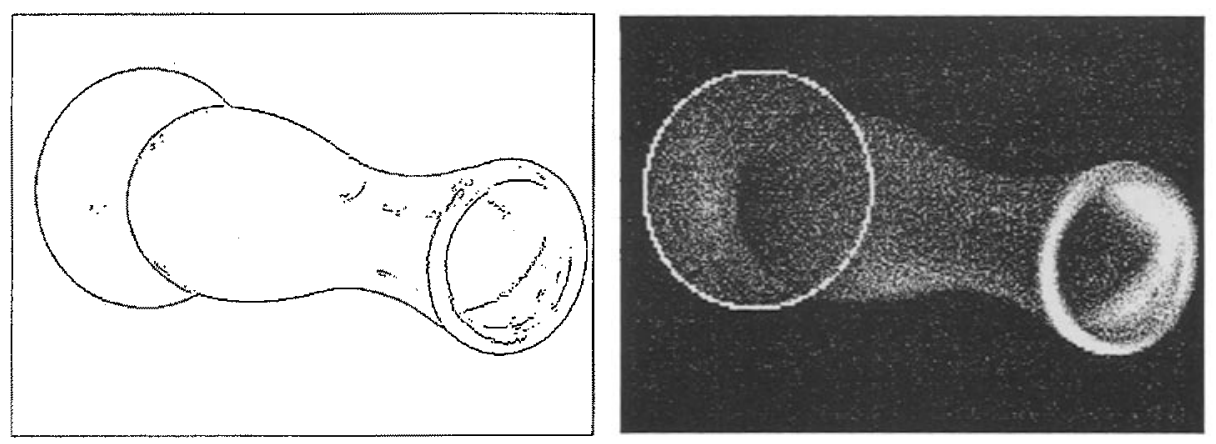

Figure 2. Detected Contours and selected detected ellipse.

A localization error occurs in the area of the top of the vase. It is due an inaccurate determination of the values of the ellipse parameters. But an iterative adjustement procedure can be used to refine the attitude. It involves matching of straight segments (straight contours after polygonal segmentation of the contour image and projections 
of the external edges of the object polyhedral model), a bucketing technique to fasten this matching, and the Lowe's algorithm [LOW-85] to calculate a new attitude after the matching. At the end of this procedure, a better attitude is obtained (figure 4).

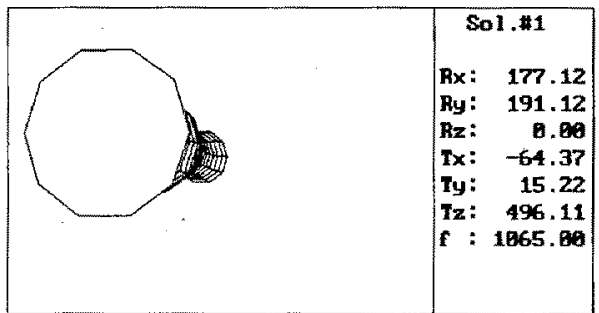

(a) score $=0.55$

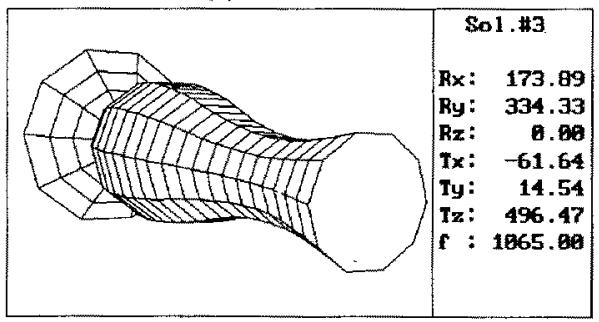

(c) score $=0.73$

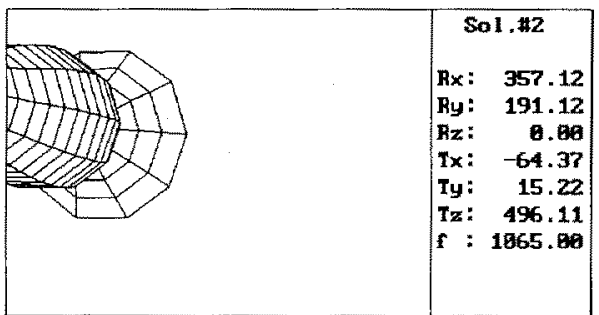

(b) score $=0.20$

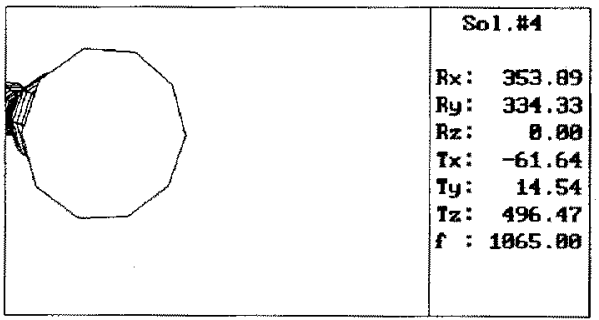

(d) score $=0.59$

Figure 3. The four compatible attitudes and the corresponding scores.
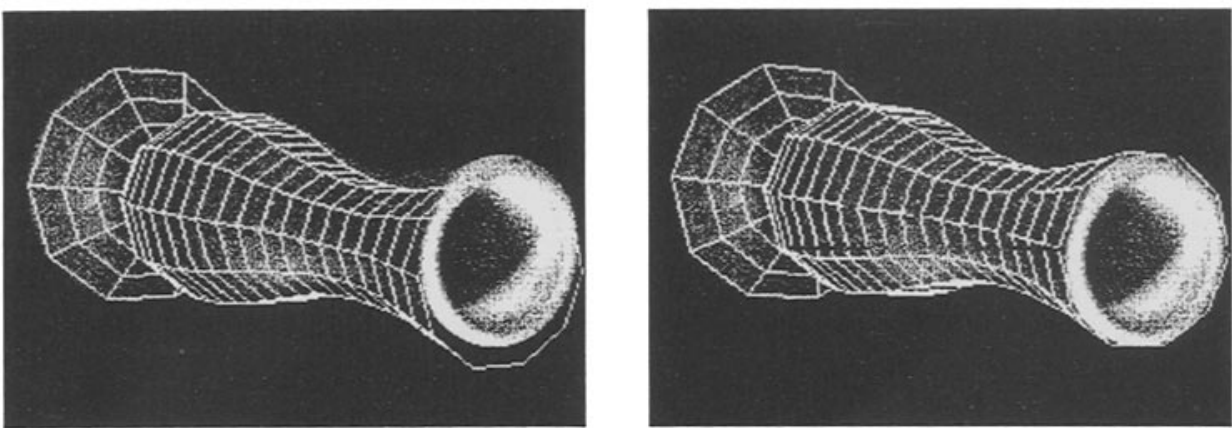

Figure 4. Selected and final model attitudes.

\section{INTERPRETATION OF ZERO-CURVATURE CONTOUR POINTS}

\subsection{Principle}

In this section, it is recalled how the zero-curvature of the contours of an object of revolution can be used to locate it.

This method relies on the following theorem [KOE-84, PON-89a] :

Theorem : The points of the limbs of an object of revolution which correspond with zerocurvature points of the scaling function of the object, are viewed under perspective projection as zero-curvature points of the contours corresponding to the projection of the limbs.

Thus if the scaling function of an object of revolution has at least one zero-curvature point and if the point of view is such that the limbs go through at least one section corresponding to a zero-curvature point of the scaling function, then the external contours of the object contains at least one pair of zero-curvature points. 
The two points of a pair and the tangents to the contours at these points define a triangle. It has been shown [RIC-89] that this triangle is the perspective projection of a cone of revolution defined by the corresponding section and by the tangents to the surface of the object at each point of this section. It has also been demonstrated that there are in general two space attitudes of the cone of revolution compatible with the observed triangle.

\subsection{Experiment}

This approach can be used for the image of figure 2.a. Figure 6 shows the selected pair of zero-curvature points on the external contours of the vase, and the tangents to the contours at these two points. The two possible attitudes of the cone of revolution are visible on figure 5. A superposition score is calculated in a same way as in the former section. The selected attitude which corresponds with the highest score leads to figure 7 on which one can see the good fit between the projection of the polyhedral model and of the brightness image.
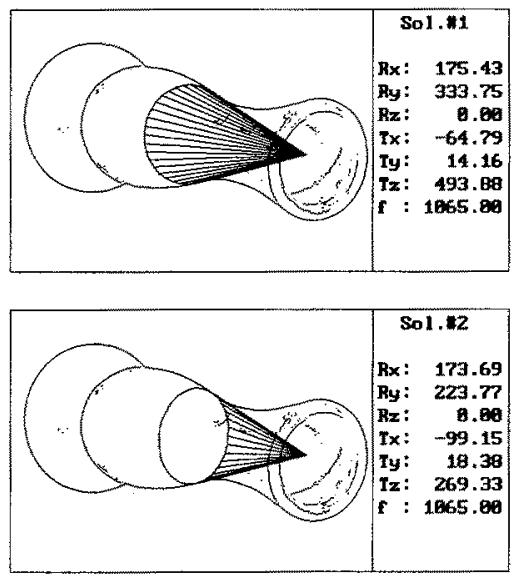

score $=0.86$

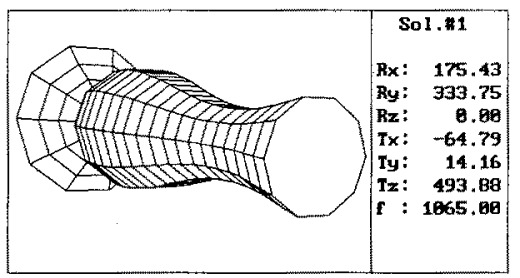

score $=0.15$

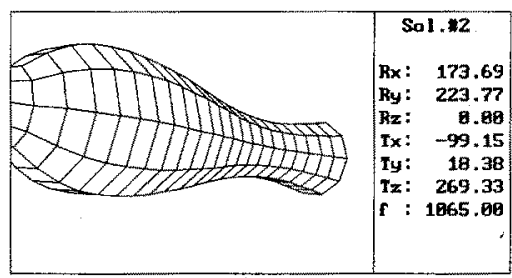

Figure 5. The two space attitudes of the cone of revolution and of the object model.

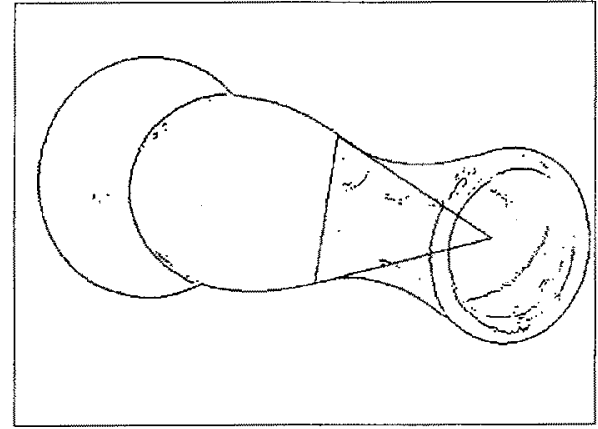

Figure 6. The triangle defined by a pair of zero-curvature contour points and the tangents to the contour at these points.

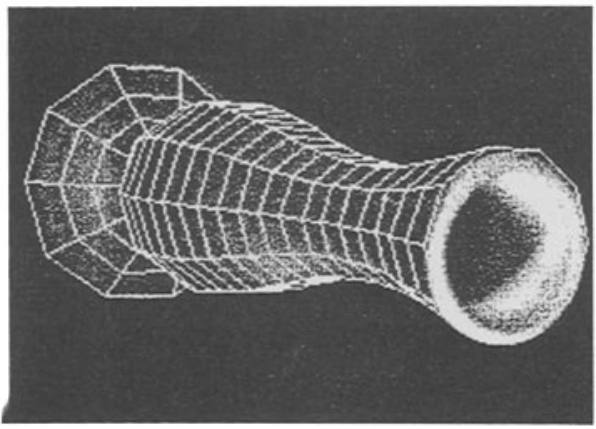

Figure 7. The superposition of the projection of the model in its calculated attitude and of the brightness image. 
The result obtained with this approach is better than the one derived from the interpretation of an ellipse. This could be surprising as it is known that it is quite difficult to locate zero-curvature points accurately. But the triangle which is interpreted is made of two segments which are the tangents to the contours at these points. Then their orientation is very precise and the triangle is not so badly defined.

\section{INTERPRETATION OF LIMBS FROM PAIRED POINTS}

\subsection{Overview}

The method presented here is a generalization of the preceding one. Using a procedure described in the next sub-section, two points of the projection of the limbs which belong to the projection of a circular section of the object are selected. As before, these two points and the tangents to the contours at these points define a triangle. But here the position of this section in the object coordinate system is supposed not to be known. So it is necessary to scan all the possible sections of the object and to calculate all the possible attitudes compatible with this triangle. In order to select the best one, a superposition score is calculated.

\subsection{Choice of a judicious viewer coordinate system}

At first, it must be noted the plane going through the optical center of the camera and the axis of revolution of the object is a plane of symmetry for the object. Thus the limbs which are situated apart this plane are symmetrical curves on the surface of the object with respect to this plane. The perspective projection of these curves are symmetrical if and only if the optical axis intersects the axis of revolution of the object.

If this is the case then the problem is much simpler since two symmetrical contour points in the image belong to the same section of the object. Fortunately it is always possible to be in this situation thanks to a transform which allows to calculate a virtual image in which the external contours are symmetrical.

For that it is necessary to know two points belonging to the same section. A pair of zero-curvature points could have been used. Here angular points have been retained. They are double points of the projection of the limbs and they can be easily obtained since the curvature of a contour reaches a maximum absolute value in a neighbourhood of these points.

Having chosen a pair of such points (see figure 8), a rotation $R_{0}$ is applied to the viewer coordinate system in order to make symmetrical the two points with respect to the vertical axis of the image (see figure 9). Transforming the projection of the limbs by this rotation leads to symmetrical external contours with respect to the vertical axis.

\subsection{Compatible attitudes}

Let us consider an object of revolution whose axis coincides with the $Z$ axis of a reference system $(O, X, Y, Z)$. The coordinates of a point $P$ of the surface of this object are $(X, Y, Z)=(r \cos \theta, r \sin \theta, Z)$ with $r=r(Z)$ being the scaling function or the generating curve of the object, and $\theta$ being the azimuth angle in the plane $(O, X, Y)$. The normal $\vec{N}$ to the surface at point $P$ is $\frac{\partial \overrightarrow{O P}}{\partial \theta} \wedge \frac{\partial \overrightarrow{O P}}{\partial z}$ and its components are $\left(n_{x}, n_{y}, n_{z}\right)=\left(\cos \theta, \sin \theta,-r^{\prime}\right)$ with $r^{\prime}$ being the derivative of $r(Z)$ with respect to $Z$.

Let us suppose also that $O$ is the optical center of the camera, that the $Z$ axis is the optical axis, and that the $X$ and $Y$ axes are respectively the horizontal and the vertical axes of the camera. 
The projection of the limbs after the rotation $R_{0}$ being symmetrical (figure 9 ), to find the space attitude of the object it is sufficient to apply to the object model a rotation $R_{\alpha}$ of angle $\alpha$ around the $X$ axis, and a translation $T_{\text {ovw }}$ of components $(0, v, w)$ to bring it in a position compatible with the observed contours.

After these two transforms, $P$ and $\vec{N}$ become $P^{\prime}$ and $\vec{N}$ respectively. They are defined by the following equations :

$$
\begin{gathered}
\left(\begin{array}{l}
X^{\prime} \\
y^{\prime} \\
Z^{\prime}
\end{array}\right)=\left(\begin{array}{ccc}
1 & 0 & 0 \\
0 & \operatorname{Cos} \alpha & -\operatorname{Sin} \alpha \\
0 & \operatorname{Sin} \alpha & \operatorname{Cos} \alpha
\end{array}\right)\left(\begin{array}{l}
X \\
Y \\
Z
\end{array}\right)+\left(\begin{array}{l}
0 \\
v \\
w
\end{array}\right)=\left(\begin{array}{c}
r \cdot \operatorname{Cos} \theta \\
r \cdot \operatorname{Cos} \alpha \cdot \operatorname{Sin} \theta-Z \cdot \operatorname{Sin} \alpha+v \\
r \cdot \operatorname{Sin} \alpha \cdot \operatorname{Sin} \theta+Z \cdot \operatorname{Cos} \alpha+w
\end{array}\right) \\
\left(\begin{array}{l}
n^{\prime}{ }_{x} \\
n^{\prime}{ }_{y} \\
n^{\prime}{ }_{z}
\end{array}\right)=\left(\begin{array}{ccc}
1 & 0 & 0 \\
0 & \operatorname{Cos} \alpha & -\operatorname{Sin} \alpha \\
0 & \operatorname{Sin} \alpha & \operatorname{Cos} \alpha
\end{array}\right)\left(\begin{array}{l}
n_{x} \\
n_{y} \\
n_{z}
\end{array}\right)=\left(\begin{array}{c}
\operatorname{Cos} \theta \\
\operatorname{Cos} \alpha \cdot \operatorname{Sin} \theta+\operatorname{Sin} \alpha \cdot r^{\prime} \\
\operatorname{Sin} \alpha \cdot \operatorname{Sin} \theta-\operatorname{Cos} \alpha \cdot r^{\prime}
\end{array}\right)
\end{gathered}
$$

Let us choose a point $p_{0}$ of the limbs projection (figure 11). Its coordinates in the viewer coordinate system are $\left(x_{0}, y_{0}, f\right)$ with $f$ being the focal length of the camera. Let $\overrightarrow{t_{0}}$ be the tangent to the contour at point $p_{0}$. Its components are $\left(a_{0}, b_{0}, 0\right)$. Let us suppose that $p_{0}$ is the perspective projection of a point $P^{\prime}$. Then equations (7), (8) and (9) hold.

$$
\overrightarrow{N^{\prime}} \cdot \overrightarrow{O p_{0}}=0 \quad \text { (7) } \quad \overrightarrow{N^{\prime}} \cdot \overrightarrow{t_{0}}=0 \quad \overrightarrow{O p_{0}}=\lambda \overrightarrow{O P^{\prime}}
$$

Combining equations (7) and (8) leads to the system of equations (10) where $c_{0}=\left(a_{0} y_{0}-b_{0} x_{0}\right) / f:$

$$
\left\{\begin{array}{ccc}
\sin \theta= & \left(\alpha_{0} r^{\prime} \cos \alpha-c_{0} r^{\prime} \sin \alpha\right) /\left(\alpha_{0} \sin \alpha+c_{0} \cos \alpha\right) \\
\cos \theta= & -b_{0} r^{\prime} /\left(\alpha_{0} \sin \alpha+c_{0} \cos \alpha\right)
\end{array}\right.
$$

But as $\cos ^{2} \theta+\sin ^{2} \theta=1$, if $t=\operatorname{tg} \alpha$, equation (11) is easily obtained from sytem (10).

$$
\left(r^{\prime 2}\left(b_{0}^{2}+c_{0}^{2}\right)-a_{0}^{2}\right) t^{2}-2 \alpha_{0} c_{0}\left(r^{\prime 2}+1\right) t+r^{\prime 2}\left(\alpha_{0}^{2}+b_{0}^{2}\right)-c_{0}^{2}=0
$$

Let us consider a particular section of the object model. For it $r(Z)$ and $r^{\prime}(Z)$ are known. Then equation (11) gives the possible values for $\alpha$. There are four determination for $\alpha$ because if $\alpha_{1}$ and $\alpha_{2}$ are solutions of (11) then $\alpha_{1}+\pi$ and $\alpha_{2}+\pi$ are solutions too.

Equation (9) gives the following system (12):

$$
\left\{\begin{array}{l}
\cos \theta=x_{0}(Z+w \cos \alpha-v \sin \alpha) / r\left(f \cos \alpha-y_{0} \sin \alpha\right) \\
\sin \theta=\left(y_{0} w-f v+\cos \alpha y_{0} Z+\sin \alpha f Z\right) / r\left(f \cos \alpha-y_{0} \sin \alpha\right)
\end{array}\right.
$$

If solutions $\alpha_{i}$ are set in equations (10) and (12), the following system is obtained which is linear in $u$ and $w$.

$$
\left\{\begin{array}{ccc}
\left(x_{0} \cos \alpha_{i}\right) w-\left(x_{0} \sin \alpha_{i}\right) v & = & r \cos \theta\left(f \cos \alpha_{i}-y_{0} \sin \alpha_{i}\right)-x_{0} Z \\
y_{0} w-f v & = & r \sin \theta\left(f \cos \alpha_{i}-y_{0} \sin \alpha_{i}\right)-\cos \alpha_{i} y_{0} Z-\sin \alpha_{i} f Z
\end{array}\right.
$$

Then rotation $R_{\alpha}$ and translation $T_{\text {ouw }}$ have been determined. It can be proved that they are at most two real possible solutions because $w$ must be positive. They are such that the projection of two points belonging to the same section (fixed $Z$ ) of the object model is compatible with the triangle in the image defined by $p_{0}, \overrightarrow{t_{0}}$ and their symmetrical with respect to the vertical axis. It must be noted that the same result as in section 3 has been obtained but with a different approach.

At this stage in order to locate the object it is necessary to find the good section. 


\subsection{Evaluation of an attitude}

For that let us choose some control points $\left(p_{1}, p_{2}, \ldots p_{i}, \ldots p_{n}\right)$ along the projection of the limbs (figure 9). For each of them, parameters $x_{i}, y_{i}, a_{i}, b_{i}$ and $c_{i}$ are known. If any particular compatible attitude $A_{j}$ is considered, its parameters $\alpha_{j}, v_{j}$ and $w_{j}$ are known too. From systems (10) and (12) the following system, linear in $Z$ and $r(Z) r^{\prime}(Z)$, can be derived:

$$
\left\{\begin{array}{rlc}
\left(a_{i} \sin \alpha_{j}+c_{i} \cos \alpha_{j}\right)\left(y_{i} \cos \alpha_{j}+f \sin \alpha_{j}\right) Z & +\left(c_{i} \sin \alpha_{j}-a_{i} \cos \alpha_{j}\right)\left(f \cos \alpha_{j}-y_{i} \sin \alpha_{j}\right) r r^{\prime} \\
& =\left(\alpha_{i} \sin \alpha_{j}+c_{i} \cos \alpha_{j}\right)\left(f v_{j}-y_{j} w_{j}\right) \\
\left(a_{i} \sin \alpha_{j}+c_{i} \cos \alpha_{j}\right) x_{i} Z & +c & \left(f \cos \alpha_{j}-y_{i} \sin \alpha_{j}\right) b_{i} r r^{\prime} \\
& = & \left(a_{i} \sin \alpha_{j}+c_{i} \cos \alpha_{j}\right)\left(v_{j} \sin \alpha_{j}-w_{j} \cos \alpha_{j}\right) x_{i}
\end{array}\right.
$$

A value $Z_{i j}$ is obtained which corresponds with the section of the object such that a limb point of this section will be projected in $p_{i}$ if the considered attitude $A_{j}$ is the good one.

The limbs equation for attitude $A_{j}$ is :

$$
\overrightarrow{O P} \cdot \overrightarrow{N^{\prime}}=r(Z)-r^{\prime}(Z) Z\left(v_{j} \sin \alpha_{j}-w_{j} \sin \alpha_{j}\right)+\sin \theta\left(v_{j} \cos \alpha_{j}+w_{j} \sin \alpha_{j}\right)=0
$$

Feeding equation (13) with $Z_{i j}$ the value of $\sin \theta_{i j}$ is obtained, and then the value of $\theta_{i j}$ since the sign of $\cos \theta_{i j}$ must be the same as the sign of the abscissa $x_{i}$ of point $p_{i}$.

Using $\theta_{i j}, Z_{i j}, \alpha_{j}, v_{j}$ and $w_{j}$, the coordinates of a point $P^{\prime}{ }_{i j}$ can be calculated. This point must be projected in $p_{i}$ if attitude $A_{j}$ is correct. But if it is not the case the projection of $P^{\prime}{ }_{i j}$ will give point $p_{i j}$ and the quadratic distance $\left|\overrightarrow{p_{i} p_{i j}}\right|^{2}$ between $p_{i}$ and $p_{i j}$ is used to evaluate the quality of the attitude $A$,

It is preferable to consider more than one point $p_{i}$ to do that. So the criterion which is used is $C(Z)=\operatorname{Min}_{j=1}^{m}\left(\sum_{i=1}^{n}\left\|p_{i} p_{i j}\right\|^{2}\right)$ where $m$ is the number of real possible attitudes for a given $Z$. Thus $m$ is equal to 2 or 1 . Let us recall that this criterion is a function of $Z, Z$ being the $Z$ coordinate of the section, in the object reference system, for which it is supposed that $P_{0}$ is the projection of one of its points. So all the values of $Z$ have to be scanned. The selected attitude of the object model is the one for which $C(Z)$ is minimal.

\subsection{Experiment}

This method has been also experimented in case of the image of figure 2 (left). Figure 8 gives the two selected angular points. After the application of $R_{0}$, symmetrical contours are obtained as shown in figure 9. On this figure, the four control points used in this experiment are also visible.

The height of the vase is nearly $250 \mathrm{~mm}$. We chose to sample the $Z$ axis each millimeter. The value of the criterion $C(Z)$ is drawn in figure 11 . Obviously this curve has two relative minima for $Z=158 \mathrm{~mm}(C(Z)=21)$ and $Z=60 \mathrm{~mm}(C(Z)=1341)$. The corresponding attitudes for the object model in the virtual image are presented in figure 10. 


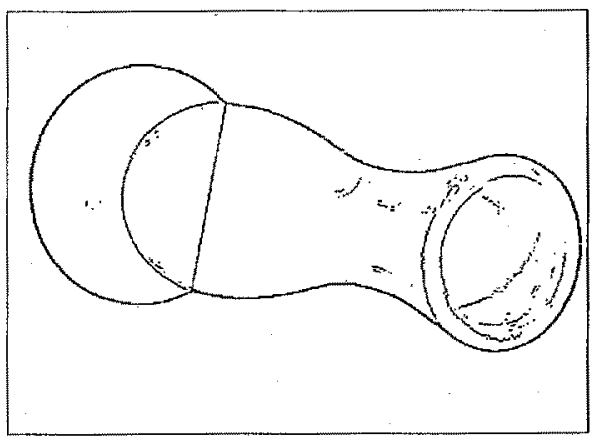

Figure 8. Selected angular contour points.

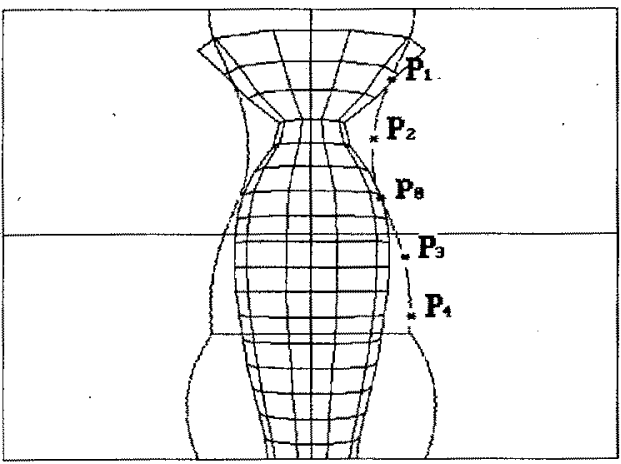

(a) criterion $=1341$

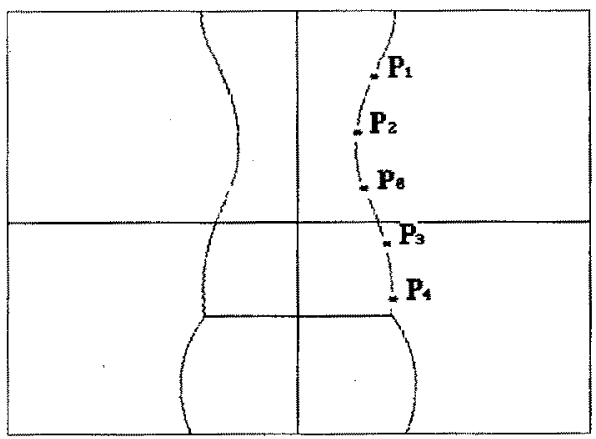

Figure 9. External contours in the virtual image.

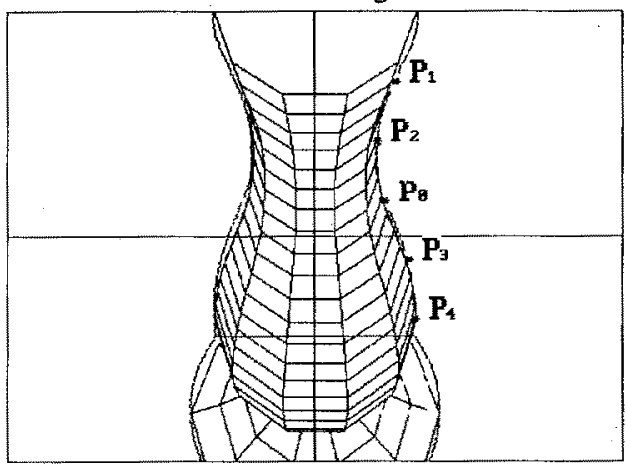

(c) criterion $=21$

Figure 10. Vase attitudes for the control point $p_{0}$ and for $Z=60$ et 158 .

After the best solution is selected, it is easy to compute the rigid transform to apply to the object model which corresponds with the interpretation of a pair of angular contour points. The superposition of the projected object model and of the brightness image is presented in figure 12 . The quality of the mutual covering of the two images is quite good. The only noticeable differences appear to be due to the polyhedral approximation.

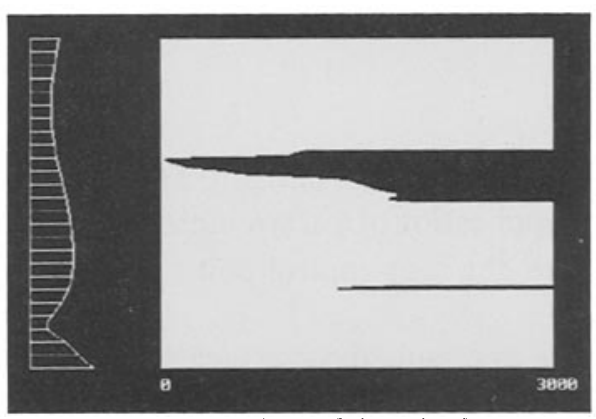

Figure 11. Values of the criterion.

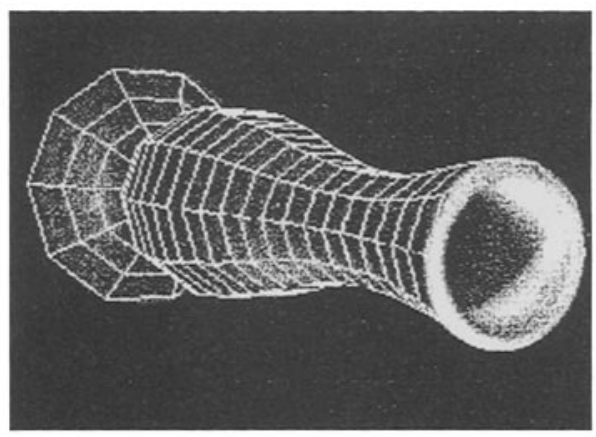

Figure 12. Projected model on the image plane. 


\section{CONCLUSION}

It has been demonstrated here how some geometrical features extracted from the brightness image of an object of revolution and coming from the perspective projection of lines or points situated on the surface of the object, can be used to find the spatial attitude of the object in the viewer coordinate system.

The various methods presented in this paper which involve different kinds of geometrical features are complementary since they could not be all present for some aspect of the object. For example, if the angle between the viewing direction and the axis of revolution is too small then elliptical contours will surely be seen meanwhile the projection of zero-curvature points of the scaling function will probably be hidden.

Before the design of an automatic system for localization of objects of revolution, the problem of the matching of similar contour points (zero-curvature or angular ones in the present case) has to be solved efficiently. So it is necessary to built a robust procedure to find the projection of the axis of revolution of these objects.

At last, it must be noted that for the three methods the localization problem is much simpler if at first the original viewer coordinate system is changed for a more judicious one. In fact with this new reference system, the direction of view always goes through the axis of revolution of the objects. It is worth noting that human vision proceeds in this way since the cone angle for accurate vision is rather small and consequently since the look is approximatively centered on the objects to be recognized or localized.

\section{BIBLIOGRAPHY}

[DH0-89] DHOME M., RICHETIN M, LAPRESTE J.T. \& RIVES G., Determination of the Attitude of $3 D$ Objects from a Single Perspective View, IEEE Trans. on PAMI, vol 11, n²12, pp1265-1278, December 1989.

[KOE-84] KOENDERINK J.J., What Does The Occluding Contours Tell Us About Solid Shape?, Perception, 13, pp321-331, 1984.

[LOW-85] LOWE D.G. Perceptual Organization and Visual Recognition, Kluwer, Boston, 1985.

[PON-89a] PONCE J., CHELLBERG D. \& MANN W., Invariant Properties of Straight Homogeneous Generalized Cylinders and Their Contours, IEEE Trans. on PAMI, vol 11, n9, pp951-966, September 1989.

[PON-89b] PONCE J.\& KRIEGMAN D.J., On Recognizing and Positioning Curved $3 D$ Objects from Image Contours, Proc. of IEEE Workshop on Interpretation of 3D Scenes, pp61-67, Austin, Tx, November 1989.

[RIC-89] RICHETIN M., DHOME M. \& LAPRESTE J.T., Inverse Perspective Transform from Zero-Curvature Curve Points. Application to the Localization of Some Generalized Cylinders, Proc. of IEEE CVPR '89, pp 517-522, San Diego, Ca, June 1989.

[SHI-87] SHIRAI Y, Three Dimensional Computer Vision, Springer-Verlag, Berlin, 1987. 